\title{
屢国 pubvet
}

https://doi.org/10.31533/pubvet.v14n9a660.1-5

\section{Dilatação vólvulo gástrica em cão de pequeno porte: Relato de caso}

\author{
Danilo do Valle Oliveira ${ }^{19}$, Ana Luiza Dias Angelo ${ }^{29}$, Aretha Alves Borges da Silva ${ }^{3 \oplus 9}$ \\ ${ }^{1}$ Residente em Clínica Médica de Carnívoros Domésticos - Universidade Federal da Bahia, UFBA, em curso. \\ ${ }^{2}$ Pós doutora em Biotecnologia pela Universidade Federal da Bahia, UFBA. \\ ${ }^{3}$ Docente do curso de Medicina Veterinária da Faculdade de Tecnologia e Ciências, FTC, Mestre em Biotecnologia pela, UFBA \\ *Autor para correspondência: E-mail: danilovoliveira@gmail.com
}

Resumo. A dilatação vólvulo gástrica é uma síndrome com alta mortalidade, acomete predominantemente cães de grande porte. Possui etiologia multifatorial, o diagnóstico é realizado através da anamnese, sinais clínicos e radiografia. A gastropexia é uma técnica cirúrgica que evita recidivas. O prognóstico é reservado, assim, a terapia precoce aumenta as chances de sobrevida do animal. Este trabalho teve como objetivo relatar os principais fatores predisponentes do vólvulo gástrico parcial em um paciente canino, Daschund, fêmea, 12 anos atendida em uma clínica veterinária particular de Salvador - Bahia.

Palavras chave: canino, gastropexia, trato gastrointestinal

\section{Dilatation gastric vulture in small dog: Case report}

Abstract. Gastric volvulus dilation is a syndrome with high mortality that predominantly affects large dogs. It has a multifactorial etiology, the diagnosis is made through anamnesis, clinical signs and radiography. Gastropexy is a surgical technique that prevents recurrences. The prognosis is reserved, so early therapy increases the animal's chances of survival. This study aimed to report the main predisposing factors of the partial gastric valve in a canine patient, Daschund, female, 12 years old attended at a private veterinary clinic in Salvador - Bahia.

Keywords: canine, gastropexy, gastrointestinal tract

\section{Dilatación vólvulo gástrico en perro pequeño: Relato de caso}

Resumen. La dilatación del vólvulo gástrico es un síndrome con alta mortalidad que afecta predominantemente a perros grandes. Tiene una etiología multifactorial, el diagnóstico se realiza mediante anamnesis, signos clínicos y radiografía. La gastropexia es una técnica quirúrgica que previene las recurrencias. El pronóstico es reservado, por lo que la terapia temprana aumenta las posibilidades de supervivencia del animal. Este estudio tuvo como objetivo informar los principales factores predisponentes de la válvula gástrica parcial en un paciente canino, Daschund, hembra, de 12 años, atendida en una clínica veterinaria privada en Salvador - Bahia.

Palabras clave: canino, gastropexia, tracto gastrointestinal

\section{Introdução}

A dilatação vólvulo gástrica (DVG) em cães é uma síndrome aguda com mortalidade de $20 \%$ a $45 \%$ mesmo naqueles que recebem tratamento. A afecção manifesta-se em duas etapas: a primeira é a dilatação gástrica e a segunda é o vólvulo (Fossum, 2014), que consiste na torção do estômago em seu próprio eixo, pode ocorrer de $90^{\circ}$ a $360^{\circ}$, mas de maneira geral acontece de $220^{\circ}$ a $270^{\circ}$. Devido ao 
ligamento gastroesplênico, ocorre deslocamento do baço para o antímero direito ventral do abdome (Ribeiro et al., 2010).

A predisposição genética tem sido relatada como fator predisponente, pois está relacionada às variações anatômicas entre esôfago e estômago, o que pode facilitar o desenvolvimento da síndrome (Feliciano et al., 2015).

A dilatação vólvulo gástrica raramente acomete cães de pequeno porte e felinos (Birchard \& Sherding, 2013).

Quanto ao sexo e ao aporte físico, macho de grande porte e/ou abaixo do peso são mais frequentemente acometidos (Willard, 2015).

Os sinais clínicos incluem êmese, anorexia, emaciação, borborígmos e timpanismo. A endoscopia normalmente não diagnostica o vólvulo crônico, é necessário a realização de radiografia (Willard, 2015). A gastropexia é a terapêutica de eleição em cães acometidos (Birchard \& Sherding, 2013; Williams \& Niles, 2014).

Como antibioticoterapia pode ser utilizado Cefazolina ( $30 \mathrm{mg} / \mathrm{kg}$ ), Amoxicilina com Clavulanato (15 a $25 \mathrm{mg} / \mathrm{kg}$ ), Enrofloxacina (5 mg/kg) ou Ampicilina $22 \mathrm{mg} / \mathrm{kg}$, associado ao Metronidazol $15 \mathrm{mg} / \mathrm{kg}$, IV (Crivellentin \& Borin-Crivelletin, 2015).

O vólvulo gástrico parcial ou crônico em cães tende a ser progressivo, mas sem risco de óbito (Birchard \& Sherding, 2013; Williams \& Niles, 2014).

O presente trabalho teve como objetivo relatar um caso clínico de vólvulo gástrico parcial em cão de pequeno porte, diagnosticado em uma clínica veterinária particular de Salvador - Bahia.

\section{Relato de caso}

Foi atendida no setor de emergência de uma clínica veterinária particular de Salvador - Bahia, com queixa de dor, uma canina da raça Daschund, $11 \mathrm{~kg}, 12$ anos. Adentrou a clínica carregada por seu tutor e defecava pastoso. A paciente apresentava-se com dor.

Durante a anamnese, o tutor referiu que o animal apresentava sialorreia, sem presença de vômito e que permaneceu prostrada, escondida e vocalizava gemidos durante o dia. Alegou que a paciente já apresentou reação adversa à Metoclopramida, que apresentava sensibilidade estomacal frequentemente e que a havia medicado com Amoxicilina com Clavulanato no mesmo dia pela manhã. Também foi relatado que a cadela é acompanhada por um ortopedista veterinário, devido a presença de hérnia de disco em segmento lombar da coluna vertebral.

Ao exame físico, observou-se mucosas hipocoradas, linfonodos não reativos, sensibilidade dolorosa à palpação da região gástrica, temperatura retal de $38,6^{\circ} \mathrm{C}$, frequência cardíaca de $200 \mathrm{bpm}$, pressão arterial sistêmica de $100 \mathrm{mmHg}$, glicemia de $76 \mathrm{mg} / \mathrm{dL}$, lactato $10 \mathrm{mmol}$ e hematócrito de $43 \%$.

A paciente foi admitida para internação por um período de 24 horas para controle da dor. Foi administrado o analgésico opioide, Cloridrato de Tramadol na dose de $4 \mathrm{mg} / \mathrm{kg}$ TID IV associado ao analgésico não opioide, Dipirona na dose de $25 \mathrm{mg} / \mathrm{kg}$ TID IV. A suspeita inicial era crise de coluna. O animal passou a noite estável, melhorou o quadro de dor aguda. Alimentou-se em pequena quantidade de ração para trato digestivo (Royal Canin i/d) e não apresentou vômito ou diarreia. Foram solicitados exames de hemograma, bioquímico (ureia, creatinina, alanina aminotransferase, fosfatase alcalina, colesterol, triglicérides, proteínas totais e frações, relação albumina/globulina), urinálise, ultrassonografia abdominal total, eletrocardiografia e ecodopplercardiografia.

Ao hemograma (Tabela 1) foi verificado diminuição de hemácias, hemoglobina e volume globular, sugestivo de anemia. Ao leucograma, constatou-se aumento dos leucócitos, bastonetes e segmentados sugestivo de processo inflamatório. Ao exame bioquímico (Tabela 2), verificou-se aumento de fosfatase alcalina, que pode ser um indicativo de congestão da veia hepática, diminuição de albumina e relação albumina/ globulina. A urinálise, apresentou aumento da densidade urinária que pode estar relacionada à retenção urinária decorrente de dor. 
No eletrocardiograma foi identificado taquicardia e um traçado compatível com sobrecarga atrial esquerda, alteração sugestiva de alteração em válvula mitral.

No ecodopplercardiograma foi observado espessamento nodular mitral compatível com processo degenerativo, insuficiência valvar mitral de grau importante, função diastólica anormal do ventrículo esquerdo (déficit de relaxamento - senilidade).

Tabela 1. Hemograma inicial da paciente canina da raça Daschund 12 anos

\begin{tabular}{lcc}
\hline Parâmetro & Resultados & Valores de Referência \\
\hline Hemácias & 5,0 & $6,0-8,0$ (milhões) \\
Volume Globular & $34 \%$ & $40-56 \%$ \\
Hemoglobina & $11,4 \mathrm{~g} / \mathrm{dL}$ & $14,0-19,0 \mathrm{~g} / \mathrm{dL}$ \\
VGM & $68,0 \mathrm{fL}$ & $65,0-78,0 \mathrm{fL}$ \\
CHGM & $33,5 \mathrm{~g} / \mathrm{dL}$ & $30,0-35,0 \mathrm{~g} / \mathrm{dL}$ \\
Plaquetas & $250,000\left(\mathrm{~mm}^{3}\right)$ & $166,000-575,000\left(\mathrm{~mm}^{3}\right)$ \\
Proteínas Totais & $6,0 \mathrm{~g} / \mathrm{dL}^{3}$ & $5,0-8,0 \mathrm{~g} / \mathrm{dL}$ \\
Leucócitos & $17,800\left(\mathrm{~mm}^{3}\right)$ & $6,000-16,000\left(\mathrm{~mm}^{3}\right)$ \\
Mielócitos & 0 & $0-0 \%$ \\
Metamielócitos & 0 & $0-0 \%$ \\
Bastões & $6 \% / 1,068$ & $0-2 \% / 0-300\left(\mathrm{~mm}^{3}\right)$ \\
Segmentados & $83 \% / 14,774$ & $55-80 \% / 3,000-11,500\left(\mathrm{~mm}^{3}\right)$ \\
Linfócitos & $10 \% / 1,780$ & $10-40 \% / 1,000-4,800\left(\mathrm{~mm}^{3}\right)$ \\
Monócitos & $1 \% / 178$ & $1-8 \% / 150-1,350\left(\mathrm{~mm}^{3}\right)$ \\
Eosinófilos & 0 & $2-10 \% / 100-1,250\left(\mathrm{~mm}^{3}\right)$ \\
Basófilos & 0 & $0-1 \% / \mathrm{raros}$ \\
\hline
\end{tabular}

$\mathrm{Na}$ ultrassonografia abdominal total, observou-se imagem sugestiva de hepatopatia, apresentou ecogenicidade diminuída; estômago acentuadamente dilatado, processo obstrutivo sugestivo de torção gástrica; baço fora de sua topografia habitual, sugestivo de uma torção esplênica parcial; nefropatia inflamatória unilateral; cistite (paredes espessas e irregulares); hemoperitônio e/ou transudato.

Tabela 2. Bioquímico inicial da paciente canina da raça Daschund 12 anos

\begin{tabular}{lcc}
\hline Parâmetro & Resultados & Valores de Referência \\
\hline Ureia & $47,0 \mathrm{mg} / \mathrm{dL}$ & $10,0-56,0 \mathrm{mg} / \mathrm{dL}$ \\
Creatinina & $1,1 \mathrm{mg} / \mathrm{dL}$ & $0,5-1,6 \mathrm{mg} / \mathrm{dL}$ \\
Alanina Aminotransferase & $48,0 \mathrm{UI}$ & $10,0-88,0 \mathrm{UI} / \mathrm{L}$ \\
Fosfatase alcalina & $381,0 \mathrm{UI} / \mathrm{L}$ & $20,0-156,0$ \\
Colesterol & $139,0 \mathrm{mg} / \mathrm{dL}$ & $100,0-275,0 \mathrm{mg} / \mathrm{dL} \mathrm{UI} / \mathrm{L}$ \\
Triglicérides & $30,0 \mathrm{mg} / \mathrm{dL}$ & $15-380 \mathrm{mg} / \mathrm{dL}$ \\
Proteínas Totais & $6,0 \mathrm{~g} / \mathrm{dL}$ & $5,4-7,1 \mathrm{~g} / \mathrm{dL}$ \\
Albumina & $1,8 \mathrm{~g} / \mathrm{dL}$ & $2,0-3,6 \mathrm{~g} / \mathrm{dL}$ \\
Globulina & $4,2 \mathrm{~g} / \mathrm{dL}$ & $2,4-5,1 \mathrm{~g} / \mathrm{dL}$ \\
Relação Albumina/Globulina & 0,4 & $0,5-1,7$ \\
\hline
\end{tabular}

Na manhã do dia seguinte o animal andou normalmente, inclusive passeou para urinar e defecar. Durante a noite do mesmo dia, a paciente foi submetida à laparotomia exploratória de emergência, devido à suspeita clínica de torção gástrica e esplênica visibilizadas ao exame ultrassonográfico. Foi visualizado estômago dilatado com antro pilórico rotacionado, baço congesto em região lateral direita e parte da curvatura do estômago com sinal de necrose. Foi realizado gastrectomia parcial da área necrótica, esplenectomia e gastropexia em parede abdominal direita. Procedimento cirúrgico realizado sem intercorrências.

A paciente foi mantida sob internação. O protocolo medicamentoso utilizado foi Cloridrato de Tramadol (4 mg/Kg TID IV) associado a Dipirona ( $25 \mathrm{mg} / \mathrm{Kg}$ TID IV). A Dipirona neste caso também 
agiu como antipirético. Empregou-se também, o antinflamatório não esteroidal (AINE), Meloxicam $0,2 \%$ na dose de $0,1 \mathrm{mg} / \mathrm{Kg}$ SID IV, protetor gástrico, o Cloridrato de Ranitidina ( $2 \mathrm{mg} / \mathrm{Kg}$ BID SC) e para a antibioticoterapia, a quinolona, Enrofloxacina (5 mg/kg BID IV) associado ao Metronidazol (15 $\mathrm{mg} / \mathrm{kg}$ BID IV). Durante a internação pós cirúrgica, a paciente apresentou-se ativa, taquicárdica, taquipneica e hipertérmica. A temperatura e o padrão respiratório retornaram à normalidade, porém a taquicardia persistiu. Ingeriu água e ração para trato digestivo (Royal Canin i/d) espontaneamente, demonstrou bastante apetite. Urinou, não apresentou disúria, dormiu durante toda a noite.

A paciente teve alta no dia 30/07/2016, porém no dia 03/08/2016 ao retornar à clínica, apresentava anorexia, no hemograma realizado foi observado leucocitose com desvio e enzimas hepáticas elevadas, foi admitida novamente para tratamento. Após $48 \mathrm{~h}$ verificou aumento dos leucócitos em relação ao último hemograma. O quadro clínico permanecia estável. Foram feitos ajustes no protocolo, retirou-se a Ceftriaxona, Enrofloxacina e o Metronidazol, introduziu o Amoxicilina com Clavulanato $(15 \mathrm{mg} / \mathrm{kg}$ BID VO) e Marbofloxacina (3 mg/kg SID VO). Após 48h de início da nova antibioticoterapia, foi realizado novamente hemograma e bioquímica sérica, constatou redução dos leucócitos e das enzimas hepáticas. A paciente foi liberada no dia 09/08/2016 para continuidade do tratamento em casa.

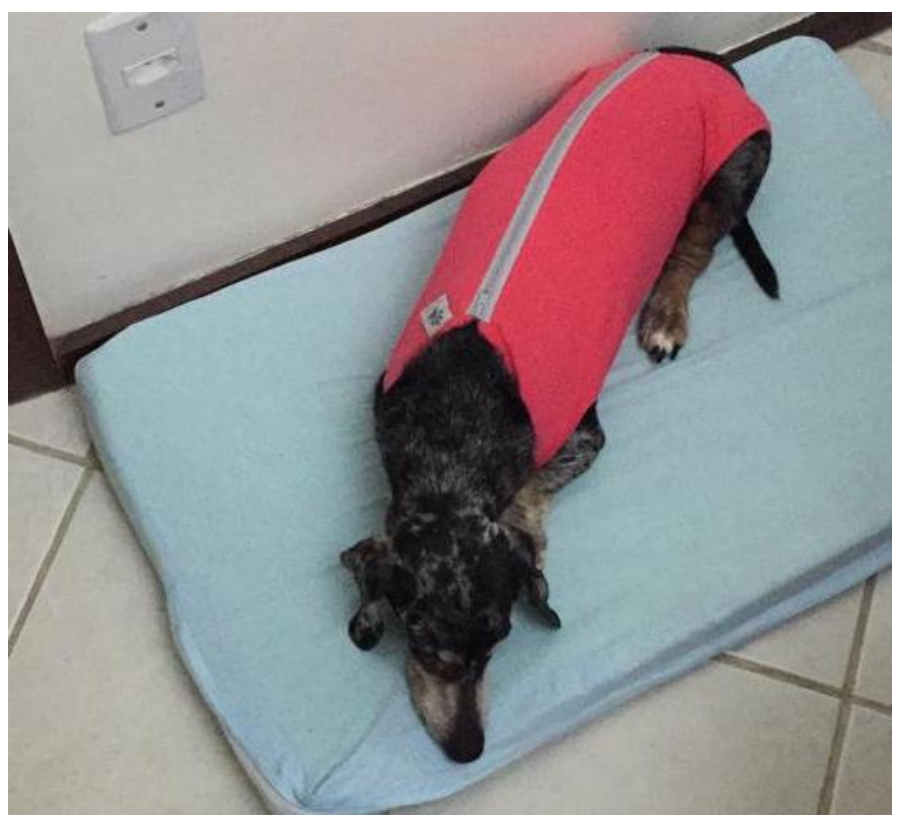

Figura 1. Paciente em recuperação pós cirúrgica na sua residência.

\section{Resultados e discussão}

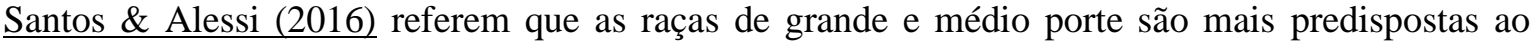
desenvolvimento da DVG. Conforme citado pelos referidos autores não é frequente a ocorrência do vólvulo gástrico em cães de pequeno porte, diferentemente do que foi evidenciado no presente relato de caso.

A radiografia é o exame de imagem de predileção para diagnosticar a DVG após estabilização do paciente (Fossum, 2014). No caso descrito o exame não foi realizado pois a suspeita clínica inicial era uma crise aguda de hérnia de disco, na qual priorizou-se a ultrassonografia abdominal devido à sensibilidade gástrica. Após a obtenção dos resultados dos exames complementares, optou-se por realizar a laparatomia exploratória de emergência com fins diagnósticos e/ou terapêuticos.

O vólvulo parcial pode ocorrer repentinamente, ser autolimitante e os cães apresentam-se clinicamente normais entre os episódios (Willard, 2015). No relato de caso, a tutora afirma que sinais clínicos como sialorreia, dor e gemido foram percebidos em outras ocasiões, um indicativo de que o animal realmente apresentava a DVG parcial.

O tratamento cirúrgico preconizado é a gastrectomia parcial do fundo do estômago, deve-se remover áreas de necrose decorrentes da DVG (Williams \& Niles, 2014), da mesma forma, no presente relato, a 
gastrectomia da área necrosada foi o tratamento instituído.

A gastropexia é a terapêutica de eleição em cães nesta condição. A técnica cirúrgica é importante para evitar recidivas, visto que fixa o estômago na parede abdominal para evitar a dilatação e consequente torção (Birchard \& Sherding, 2013; Williams \& Niles, 2014). Esta técnica cirúrgica foi utilizada na paciente descrita no relato de caso.

A antibioticoterapia utilizada no presente relato caso está de acordo com a literatura recomendada para tratamento de infecção decorrente da DVG (Crivellentin \& Borin-Crivelletin, 2015).

\section{Conclusão}

A paciente foi submetida a terapia de suporte e laparatomia exploratória com fins diagnósticos e terapêuticos. A DVG não ocorre com frequência em cães de pequeno porte, portanto, é preciso realizar maiores estudos sobre a afecção nestes animais.

\section{Referências bibliográficas}

Birchard, J. S., \& Sherding, R. G. (2013). Doenças do Estômago. Birchard \& Sherding. Manual Saunders de Clínica de Pequenos Animais. 3. Ed. São Paulo., 29.

Crivellentin, L. Z., \& Borin-Crivelletin, S. (2015). Casos de rotina em medicina veterinária de pequenos animais. In MedVet.

Feliciano, M. A. R., Canola, J. C., \& Vicente, W. R. R. (2015). Faringe, Esôfago e Estômago. In: Maronezi et al. Diagnóstico por Imagem em Cães e Gatos. São Paulo: MedVet.

Fossum, T. W. (2014). Cirurgia de pequenos animais (4th ed., Vol. 1). Elsevier Brasil.

Ribeiro, L., Cabrini, T. M., Friolani, M., \& Dias, L. G. G. G. (2010). Síndrome da dilatação vólvulo gástrica em cães-relato de caso. Revista Científica Eletrônica de Medicina Veterinária. São Paulo, $15,1-7$.

Santos, R., \& Alessi, A. (2016). Sistema Digestório. In Patologia Veterinária. 2. ed. Rio de Janeiro. (pp. 487-572).

Willard, M. D. (2015). Doenças do Estômago. In: Nelson, R. W. \& Couto, C. G. Medicina interna de pequenos animais. 5 . ed. Rio de Janeiro.

Williams, J. M., \& Niles, J. D. (2014). O estômago. In: Ellison, W. G. BSAVA Manual de Cirurgia Abdominal em Cães e Gatos. São Paulo.

Recebido: 10 de maio, 2020.

Aprovado: 19 de junho, 2020.

Disponível online: 14 de setembro, 2020

Licenciamento: Este artigo é publicado na modalidade Acesso Aberto sob a licença Creative Commons Atribuição 4.0 (CC-BY 4.0), a qual permite uso irrestrito, distribuição, reprodução em qualquer meio, desde que o autor e a fonte sejam devidamente creditados. 